\title{
Aportes de la Sociología de la Educación la formación de profesores de educación primaria
}

\section{Contributions of the Sociology of Education the training of primary education teachers}

DOI: http://dx.doi.org/10.17981/cultedusoc.12.1.2021.19

Recibido: 21 de enero de 2020. Aceptado: 10 de septiembre de 2020. Publicado: 10 de febrero de 2021

José Eriberto Cifuentes-Medina (i)

Universidad Pedagógica y Tecnológica de Colombia. Tunja (Colombia)

joseeriberto.cifuentes@uptc.edu.co

Nubia Elena Pineda-de-Cuadros (1)

Universidad Pedagógica y Tecnológica de Colombia. Tunja (Colombia)

nubia.pineda@uptc.edu.co

Jaime Andrés Torres-Ortiz (D)

Universidad Pedagógica y Tecnológica de Colombia. Tunja (Colombia)

Jaime.torres@uptc.edu.co

Para citar este artículo:

Cifuentes-Medina, J., Pineda-de-Cuadros, N. \& Torres-Ortiz, J. (2021). Aportes de la Sociología de la Educación la formación de profesores de educación primaria. Cultura, Educación y Sociedad, 12(1), 297-310. DOI: http://dx.doi.org/10.17981/ cultedusoc.12.1.2021.19

\section{Resumen}

En el presente artículo se analizan y comparan desde una dimensión teórica-conceptual los aportes dados por teóricos de la Sociología en sus inicios históricos, en relación con los referentes contemporáneos de la Sociología de la Educación, evidenciando puntos de tensión y discusión situados principalmente entre la sociología funcionalista crítica y progresista, con énfasis en esquemas conceptuales con relaciones y diferencias de orden histórico, económico y cultural. La relación se configura en un escenario el cual, hasta el presente, demuestra que la trascendencia de referentes históricos y sociológicos, inciden en las líneas teóricas de la sociología de la educación, entre estos, el Marxismo y la teoría sociológica de Max Weber.

Palabras clave: Sociología; educación; formación de profesores; educación básica

\section{Abstract}

In this article, the contributions given by theorists of Sociology in its historical beginnings are analyzed and compared from a theoretical-conceptual dimension, in relation to contemporary referents of the Sociology of Education, evidencing points of tension and discussion located mainly between the Critical and progressive functionalist sociology, with an emphasis on conceptual schemes with relationships and differences of historical, economic and cultural order. The relationship is configured in a scenario that, up to the present, shows that the transcendence of historical and sociological references affects the theoretical lines of the sociology of education, among them, Marxism and the sociological theory of Max Weber.

Keywords: Sociology; education; teacher training; basic education 


\section{INTRODUCCIÓN}

El análisis parte de las generalidades de la Sociología (Araque y Rivera, 2005) avanzando en los fundamentos (Rodríguez, 2011) y la Sociología de la Educación (Brigido, 2016) y llegando a los aportes específicos al estudio de la Educación desde (Rojas-León, 2014) en la visión de varios autores clásicos y la proyección de los nuevos enfoques de la sociología y tendencias de una disciplina que ha delimitado su objeto de estudio en la educación y en qué medida puede contribuir a la formación de profesores de educación básica primaria.

La formación de los profesores ha de ser permanente, no solamente en el desarrollo del pensum académico durante su proceso de preparación a la obtención del título profesional de licenciados otorgado por la universidad; sino también de una formación continua en una línea de formación disciplinar o también en escenarios transversales, como es la sociología de la educación, que permite a los docentes en efecto trabajar una posible relación entre el acto pedagógico en el marco de la escuela y el proceso educativo en la sociedad para comprender el posible ambiente heterogéneo de cada niño.

\section{Fundamentación Epistemológica del objeto de estudio}

En el desarrollo temático se infiere el análisis de una hermenéutica inicial de concepción de Sociología y Sociología de la Educación al igual que de las corrientes sociológicas que han estudiado el fenómeno de la educación en sus aspectos generales para consolidar el objeto de análisis en el presente artículo. En el inicio de la connotación objetiva, teniendo en cuenta la posibilidad de la presencia del proceso del debate epistemológico de educación, sociología y sociología de la educación, a través del estudio de las corrientes sociológicas y el estudio de la educación y del sistema escolar. Se realiza un esbozo de la definición epistemología de sociología, educación y sociología de la educación para avanzar en el proceso de reflexión sistemática. Desde este referente se presentan las principales concepciones disciplinares en la Tabla 1.

TABLA 1.

Definición etimológica de Sociología y Educación

Sociología

Educación
Sociología de la educación
Es el estudio científico de la vida social. Es una ciencia que estudia los sucesos de la gente, el público tiende a considerar que la sociología es sólo sentido común. Suele decirse que los sociológicos estudian cosas que todo el mundo sabe.
Procede del termino latino educare, cuyo significado es: "criar, alimentar o instruir".Y Educere significa el promover al desarrollo intelectual del educando. Es el proceso de facilitar el aprendizaje, conocimientos, habilidades, valores, creencias y hábitos de un grupo de personas que los transfieren a otras a través de diversos métodos.
Es una perspectiva para el análisis del fenómeno educativo que utiliza los conceptos, metodologías y teorías de la sociología para entender la educación en su dimensión social. Como perspectiva se ha nutrido de aportes de sociólogos, pedagogos, psicólogos, antropólogos y economistas, constituyéndose como un campo interdisciplinario en Educación. 
Una vez se esgrime la procedencia etimológica, epistemológica y su significado se avanza en el análisis hermenéutico de la sociología y sobre todo del objeto de estudio que corresponde a la Sociología de la Educación, a decir de Brigido (2016):

Por tratarse de una sociología especial, el área de la Sociología de ale educación es un área propia de la sociología, de amanera que sus problemas se tratan como problemas sociológicos y no como problemas de practica pedagógica ni problemas sociales.... El aparato conceptual que fija la perspectiva de análisis de esa problemática, es sociológico. Esto implica que quien pretenda hacer investigación en sociología de la educación, debe previamente conocer y manejar adecuadamente ese marco conceptual. Los métodos y técnicas de investigación que utiliza la sociología de la educación son los mismos que utiliza la sociología, y la lección de los más adecuados para un paso concreto depende del marco teórico adoptado para la investigación (p. 45).

El propósito de la fundamentación etimológica y epistemológica contribuye a fortalecer el objeto de estudio y en adelante consolidar el corpus teórico a debatir en el proceso de formación de profesores en educación básica primaria, como fundamento para el estudio del acto educativo desde la sociología de la educación y su apropiación en el contexto de desempeño profesional.

Se realiza desde un enfoque de investigación cualitativa a través de un análisis documental, en la cual se recopila y selecciona información a través de la lectura de documentos, libros, revistas, artículos que sirven de insumo para la síntesis, análisis e interpretación de los documentos en el marco de una investigación informativa.

\section{Generalidades de la Sociología de la Educación}

En este aparte se reconoce la definición de Sociología desde el punto de vista de dos autores de mediados del siglo XX: Adorno y Horkheimer (1969). Una vez expuesta la etimología se avanza a la definición sustentada desde Rojas-León (2014) y Brigido (2016) en el propósito de consolidar la connotación del estatus epistemológico de la Sociológica desde los autores y el desarrollo de su corpus propio de una ciencia y la Sociología de la Educación como disciplina o rama que nace de la Sociología, también con su propio corpus ontológico y epistemológico (Tabla 2).

La Sociología de la Educación, no se reduce a los problemas de la Escuela, sino que abarca el ámbito donde tiene lugar un proceso educativo o de socialización e incluye, de manera particular, la problemática relativa a la compleja relación que se da entre el proceso de educar y la sociedad en su conjunto. Por lo tanto, es pertinente el fortalecimiento de las nociones generales y la cimentación del estudio del objeto por parte de la sociología de la educación en escenarios de educación básica primaria.

\section{Aproximación a las corrientes sociológicas}

Entre las corrientes sociológicas que han estudiado el fenómeno de la educación, según Bernal (2006) se encuentran las siguientes: La primera corresponde a la funcionalista de la Sociología de la Educación, el debate entre educación y empleo de los años cincuenta e inicio de los sesentas, la segunda corresponde al conflicto y reproducción en la Sociología 
TABLA 2.

Sociología y el objeto de la Sociología de la Educación

\begin{tabular}{|c|c|}
\hline Aspecto & Breve descripción \\
\hline ¿Qué es la sociología? & $\begin{array}{l}\text { La sociología, según Adorno y Horkheimer (1969), nace como "hija } \\
\text { del positivismo" y, desde sus inicios, busca liberar a la voluntad y } \\
\text { el conocimiento de la influencia del saber religioso y de cualquier } \\
\text { especulación metafísica proveniente de la filosofía, por medio de la } \\
\text { rigurosidad y objetividad de la investigación científica aplicada a la } \\
\text { comprensión de los fenómenos sociales. } \\
\text { La definición ofrecida por sí misma no permite comprender en } \\
\text { toda su extensión a la sociología como ciencia y sus posibles } \\
\text { repercusiones en la realidad social. Para lograr esto es necesario } \\
\text { profundizar en las diversas perceptivas o tradiciones teóricas. En } \\
\text { cuanto a su utilidad, este es un tema de debate que depende de la } \\
\text { posición asumida, tanto de quien pregunta como de quien contesta } \\
\text { y que sólo el lector podrá juzgar cuando profundice, por su cuenta, } \\
\text { en la producción sociológica. }\end{array}$ \\
\hline $\begin{array}{l}\text { ¿Cuál es el objeto de estudio de la } \\
\text { Sociología de la Educación? }\end{array}$ & $\begin{array}{l}\text { Autores como Durkheim consolidad la educación como el } \\
\text { curriculum, tanto oficial como oculto, debido a que se enfoca tanto } \\
\text { en los contenidos como en los valores morales. En cambio, para } \\
\text { Weber la educación interviene en la sociedad por medio de los } \\
\text { estamentos. Por otro lado, Weber también se enfoca en el tema } \\
\text { del poder detrás del nombramiento de profesores universitarios y } \\
\text { sostiene que inevitablemente las influencias políticas condicionan } \\
\text { el avance o estancamiento de algunos. } \\
\text { En cambio, a Marx le interesa destacar el factor ideológico de } \\
\text { la educación detrás de la dominación y la reproducción de las } \\
\text { condiciones de producción y la marginalidad que sufre la clase } \\
\text { proletaria respecto a las condiciones en las que se desarrolla su } \\
\text { educación. Simmel por su parte centra su atención en el proceso de } \\
\text { enseñanza y aprendizaje, el papel del docente, la metodología en las } \\
\text { lecciones, etc., y en el acontecer de la lección y la educación. }\end{array}$ \\
\hline
\end{tabular}

Fuente: Información recabada de Rojas-León (2014) y Brigido (2016).

de la Educación, y la tercera atañe a la Sociología Interpretativa de los años 80, es la Sociología de la Educación "interpretativa" de Young, la teoría de la resistencia de Willis y Giroux, y los estudios de Género y Etnia, al final de los ochenta.

En ese orden, el planteamiento epistemológico no se profundiza, pero es motivo de discusión en el campo de las ciencias de la educación. Además, concibe que la Sociología de la Educación no se reduce a solo los problemas de la escuela en el acto pedagógico, sino que abarca cualquier ámbito donde tiene lugar el proceso educativo e incluye, de manera particular la problemática relativa a la compleja relación que se da entre ese proceso y la sociedad en su conjunto e integra lo que Durkheim denominó Ciencia de la Educación. Para Durkheim, según Austin (s. f.) afirma:

Sólo de esta manera pueden quedar garantizadas la cohesión y la supervivencia de la sociedad. Al mismo tiempo que Durkheim exponía su visión sociológica de la educción creó una especialidad, la de la sociología de la Educación, que es un campo de la sociología que se encarga de evaluar los procesos educativos en las sociedades modernas (p. 2). 
La Sociología de la Educación tiene la misión de evaluar los alcances y el impacto del proceso de educar en una sociedad moderna, por tanto, es necesario que los profesores del siglo XXI puedan poseer conocimientos de este campo de la sociología desde el siglo XX en estudios realizados por Austin (s. f.) y Bonal (1998). La Tabla 3 evidencia lo planteado.

TABLA 3.

Etapas en el desarrollo de la Sociología de la Educación

\begin{tabular}{cc}
\hline Corriente & Breve Descripción \\
\hline
\end{tabular}

Durante los años 60, cobró importancia la preocupación por examinar y comparar los altos y bajos rendimientos que demostraban niños y jóvenes en la educación: altos para las clases medias y superiores y deficitarias para las clases bajas. A

La corriente funcionalista de la sociología de la educación. El debate entre educación y empleo. (Años cincuenta, inicio de los sesenta).

\section{Conflicto y reproducción en}

la sociología de la educación

(Década de los sesenta e inicios de los setenta).

La vertiente de la reproducción cultural.

La vertiente marxista.

Althusser y la escuela como Aparato Ideológico del Estado

Boudelot y Establet. Teoría de la correspondencia. Wobles y Gintis y la teoría de la correspondencia.

La sociología Interpretativa de los años 80. La sociología de la educación "interpretativa" de M. Young.

La teoría de la resistencia de Willis y Giroux, y los estudios de Género y Etnia, al final de los ochenta. partir de esta preocupación se abrieron numerosas nuevas perspectivas que han estado examinando completamente el proceso educativo, a través de sus tres procesos básicos —al decir de O. Banks-el programa, la pedagogía del aula y la evaluación. Con el correr del tiempo se han desarrollado muchas otras perspectivas sociológicas del papel que tiene la educación en la reproducción y formación de la sociedad.

Dos vertientes o corrientes teóricas son las que caracterizaron este periodo, por un lado, una actitud epistemológica ecléctica que combina a Marx, Durkheim/Parsons, Mead y otros pensadores. Por otro lado, una actitud renovadora del marxismo europeo de los sesenta y setenta en las tesis neomarxistas que desde distintos matices teóricos ven a la educación como un instrumento para la preservación del poder, la división de clases y el capitalismo occidental. La más famosa y conocida de estas tesis fue la de Althusser y su "concepción de la educación como un aparato ideológico del Estado", acompañados por la teoría de la doble red de Boudelot y Establet y la teoría de la correspondencia de Bowles y Gintis.

Esta línea de investigación teórica y empírica se ajusta estrictamente a la dirección que, según su Young, debió tomar la nueva sociología de la educación a partir de los años setenta: desenmascarar la construcción social del conocimiento educativo. Frente a una sociología funcionalista de la educación centrada en los procesos de socialización y selección (objetos que también son centrales en las teorías de la reproducción), el 'paradigma interpretativo' considera que 'la sociología de la educación ya no es concebible como un área de investigación distinta de la sociología del conocimiento' (Young, 1971) y reorienta la investigación hacia la interacción entre profesor y alumnado, las categorías o conceptos utilizados por los educadores y el currículum" (...). 
En la perspectiva de Austin (s. f.) retomando a Bonal (1998), señala:

Una perspectiva muy popular en la sociología de la educación hasta fines de los años 60 fue el funcionalismo y procede fundamentalmente de los trabajos del francés Durkheim. El sistema funcionalista, tal como lo emplea Durkheim, consiste en buscar la función desempeñada por una institución (la educación en nuestro caso) en la sociedad en general; es decir, el rol que juega esa institución en la promoción y el mantenimiento de la cohesión y de la unidad sociales. Todas las grandes instituciones estudiadas por Durkheim se hallan concebidas de esta forma, y la educación no es una excepción. Alude así al desarrollo en el niño de ciertos valores y de determinadas destrezas intelectuales y físicas necesarias para convertirse en parte de la sociedad y que le son traspasadas por la institución educacional (p. 41).

Las etapas que ha desarrollado, estudiado y cimentado Bonal (1998), en su obra "Sociología de la Educación: Una aproximación crítica a las corrientes contemporáneas", en el recorrido por la segunda mitad del siglo XXI, se centra en las tres etapas anteriormente expuestas e incluso fija una posición del escenario actual de la Sociología de la Educación, considerando los cambios actuales de la sociedad y la educación. Realidad susceptible de contradicción según el contexto particular pero posible de afirmar en un escenario general.

\section{El supuesto funcionalista en la sociología de la educación}

La mayoría de la sociología de la educación crítica es una simple inversión de la sociología funcionalista clásica en su explicación del sistema escolar, asegura Criado (2003), quien además afirma:

El supuesto funcionalista, aplicado al sistema escolar, supone una premisa fundamental del análisis: El sistema escolar es un instrumento que se explica por sus funciones sociales El sistema escolar es un instrumento que se explica por sus funciones sociales. Es un punto fundamental del funcionalismo clásico: para Parsons y Durkheim, la escuela es un órgano especializado de la sociedad que asegura la cohesión social y la formación apropiada a la división del trabajo (p. 2).

Algunos sociólogos, consideran la sociología como un instrumento de las clases dominantes para perpetuar la dominación capitalista: ya sea aparato ideológico del Estado, otra institución disciplinaria o un sistema escolar aparentemente autónomo, pero realmente dependiente.

La hipótesis central concibe lo siguiente:

[...] se comparte también el supuesto de que la escuela consigue sus objetivos y de que lo hace, fundamentalmente, por su forma de organización Parsons, autores marxistas, Bourdieu y Passeron, Foucault y por la autoridad pedagógica del maestro ya sea debida a convicción interior -Durkheim- o a la delegación de las clases dominantes-Bourdieu y Passeron (Criado, 2003, p. 4).

La hipótesis admite el análisis en el sistema escolar como conjunto internamente integrado con las instituciones externas; la historia del sistema escolar se explica por sus funciones. Al igual que el estudio del acto educativo como se explica en la Tabla 4 para develar las corrientes y precisar su descripción. 
TABLA 4.

El supuesto funcionalista en la sociología de la educación crítica

\begin{tabular}{|c|c|}
\hline Corriente & Breve descripción \\
\hline $\begin{array}{l}\text { El sistema escolar } \\
\text { como conjunto } \\
\text { internamente } \\
\text { integrado. }\end{array}$ & $\begin{array}{l}\text { La escuela puede cumplir sus funciones porque su organización interna no supone } \\
\text { estructuras en tensión: cada parte del sistema se explica por la contribución que } \\
\text { realiza al mantenimiento de éste. En Parsons (1976), las distintas etapas de la } \\
\text { escolarización tienen su función específica en la socialización en valores universales y } \\
\text { en la especialización. }\end{array}$ \\
\hline $\begin{array}{l}\text { El sistema escolar } \\
\text { como conjunto } \\
\text { armoniosamente } \\
\text { integrado con } \\
\text { las instituciones } \\
\text { externas. }\end{array}$ & $\begin{array}{l}\text { Si la escuela puede cumplir perfectamente sus funciones sociales, es porque no } \\
\text { se halla en tensión con otras instituciones, sino en relación armoniosa, funcional. } \\
\text { Así, con el mercado de trabajo: la escuela aseguraría la formación de trabajadores } \\
\text { especializados, aunque lo fundamental no fuera tanto la cualificación técnica como la } \\
\text { inculcación de una serie de hábitos y normas morales -Parsons, Althusser, Bowles y } \\
\text { Gintis. Con la estratificación social: la escuela aseguraría la distribución de personas } \\
\text { en las posiciones de la manera más funcional: de acuerdo a mérito -Durkheim, } \\
\text { Parsonso reproduciendo de manera legítima la estructura de clases -Althusser, } \\
\text { Baudelot y Establet, Bowles y Gintis, Bourdieu y Passeron. }\end{array}$ \\
\hline $\begin{array}{l}\text { La historia del } \\
\text { sistema escolar } \\
\text { se explica por sus } \\
\text { funciones. }\end{array}$ & $\begin{array}{l}\text { La historia del sistema escolar es la historia de su avance hacia el mejor } \\
\text { cumplimiento de sus funciones. Esta idea es la que mueve el análisis de Durkheim } \\
\text { en La evolución pedagógica en Francia: hay una evolución natural de la escuela } \\
\text { esporádicamente trastornada por desarrollos patológicos. Para los autores marxistas, } \\
\text { la historia de la escuela es la historia de cómo la burguesía la ha ido conformando a } \\
\text { sus intereses. }\end{array}$ \\
\hline
\end{tabular}

Fuente: Información recabada de Criado (2003).

La escuela sería el instrumento de la sociedad para conseguir un orden social justo mediante la socialización metódica de los niños. Este discurso es de Durkheim y Parsons, y son estas ideas las que se hallan en el centro de las reformas escolares del siglo XX. Premisa, que hace parte de la hipótesis en estudio y contribuye a la discusión de la sociología de educación citica desde la perspectiva de Criado (2003). Los procesos de educación no siempre son consecuentes según los contextos y las particularidades de los sujetos del acto educativo por lo tanto las reformas estandarizadas no siempre generan resultados esperados, dado que el sistema se proyecta en sentido homogéneo donde muchas veces se desconocen las particularidades heterogéneas del contexto, la relación del acto educativo y de los sujetos inmersos. Por ello, Rodelo y Castro (2016), enfatizan en la necesidad de que la educación, a través de la escuela y el acto educativo, contribuyan a fortalecer el desarrollo humano y social de los individuos.

\section{Retórica de las funciones en el sistema escolar}

De hecho, se puede decir que todas las explicaciones funcionalistas parten de un recurso retórico fundamental: convertir el efecto en función del mecanismo base de la explicación funcionalista, por lo tanto, los efectos de las instituciones se convierten en funciones de estas, sin una reflexión cimentada de retrospección y proyección. Criado (2003) afirma que, "el sistema escolar sería un aparato destinado a producir ese efecto o un mecanismo milagrosamente ajustado (la expresión es de Bourdieu y Passeron) para producirlo" (p. 13). Aspectos que conllevan a una posible determinación retórica de los efectos y funciones como se expone en la Tabla 5 para documentar los tres dimensiones objeto de estudio. 
TABLA 5.

Retórica de los efectos o funciones

\begin{tabular}{|c|c|}
\hline Aspecto & Breve descripción \\
\hline En primer lugar. & $\begin{array}{l}\text { No todos los efectos del sistema escolar se equiparán } \\
\text { a funciones. Y ello porque este esquema se combina } \\
\text { en la explicación con otro esquema básico: la } \\
\text { distinción entre buenos y malos. Los «buenos» son } \\
\text { la clase obrera, las mujeres, las minorías étnicas } \\
\text { o incluso los niños. Los «malos» son las clases } \\
\text { dominantes o la burguesía y sus aliados. Este } \\
\text { esquema es básico para proporcionar explicaciones } \\
\text { «críticas». }\end{array}$ \\
\hline En segundo lugar. & $\begin{array}{l}\text { El concepto de función permite convertir las } \\
\text { descripciones en explicaciones sin analizar con } \\
\text { detenimiento las dinámicas que producen el } \\
\text { efecto. Vemos un efecto, constatamos que es malo, } \\
\text { y lo explicamos: la función del sistema escolar es } \\
\text { producirlo. De esta manera, aparte de ahorrarnos } \\
\text { trabajo -describir con minucia las dinámicas que } \\
\text { lo producen, sus condiciones de posibilidad, las } \\
\text { condiciones en que se modifican en algún sentido } \\
\text { los efectos-, nos podemos presentar en público como } \\
\text { críticos inteligentes, se ha descubierto, tras la } \\
\text { aparente bondad del sistema, su maldad intrínseca. }\end{array}$ \\
\hline El tercer lugar. & $\begin{array}{l}\text { A destacar de la sustitución de «efecto» por } \\
\text { «función» es que supone, en la mayoría de los casos, } \\
\text { un realismo de las instituciones: éstas serían } \\
\text { mecanismos destinados a cumplir una función; más } \\
\text { aún, serían las instituciones los sujetos de la acción: } \\
\text { el sistema escolar apañaría todos los elementos para } \\
\text { cumplir su función. }\end{array}$ \\
\hline
\end{tabular}

Fuente: Información recabada de Criado (2003).

Al pasar de los efectos a funciones se presenta, sin embargo, un problema cuando se plantea desde una perspectiva crítica. Se puede comparar con el funcionalismo clásico: éste, al analizar el sistema escolar, toma al pie de la letra los discursos de los reformadores escolares: la función de la escuela es promover la igualdad de oportunidades.

En el análisis de la crítica de la Sociólogia de la Educación a decir de Criado (2003) considera:

El pensamiento crítico en torno a la escuela ha estado girando durante demasiado tiempo en la búsqueda de funciones de un tipo u otro. En este sentido, ha sido poco más que una inversión de los esquemas del funcionalismo clásico. En vez del análisis detenido de las dinámicas que se producen en el seno de los sistemas escolares, de sus condiciones de producción y posibilidad, de los efectos diferenciales que generan en distintas configuraciones históricas, se ha dedicado, bajo el lema "nadie a mi izquierda», a competir por demostrar que el sistema es el peor de los posibles, esto es, a demostrar que todo está atado y bien atado para mayor gloria de las clases dominantes. El funcionalismo está tan profundamente enraizado en los esquemas cognitivos de los sociólogos «críticos», que cualquier crítica al mismo puede ser rápidamente descalificada como reaccionaria (p. 22). 
El argumento de Criado (2003) es un posible obstáculo a la conformación de una sociología de la educación verdaderamente "alternativa», esto es, a una Sociología de la Educación que sea capaz de proponer alternativas a la vez progresistas y realistas, de acuerdo con los contextos y realidades del sistema educativo y del estudio del fenómeno educativo.

\section{Aportes de la Sociología al estudio de la educación: autores clásicos}

Las tradiciones teóricas que refieren el estudio de la educación son numerosas y los aportes que desde la sociología se han desarrollado acerca de la educación son significativos para entender las implicaciones en el proceso hermenéutico del acto educativo.

Los aportes se podrían organizar por escuelas, tradiciones o corrientes para un mayor proceso interpretativo, explicativo y comprensivo del tema y de las diversas perspectivas dentro de una misma ciencia. Desde la perspectiva de Brigido (2016) y Rojas-León (2014), en este apartado se analiza la forma en que los máximos representantes de la sociología clásica: Karl Marx (1818-1883), Max Weber (1864-1920), Emile Durkheim (1858-1917) y Georg Simmel (1858-1918), reflexionaron sobre la educación.

La forma como ellos han abordado este fenómeno, aunque no de forma explícita, ha dado origen a la rama de la sociología denominada Sociología de la Educación. Esta disciplina nace, prácticamente, con la propia sociología, a pesar de que su institucionalización se da como tal a mediados del siglo XX. De los cuatros autores clásicos, quien se abocó al estudio de la educación de manera explícita fue Durkheim. Al respecto, Brigido (2016) afirma que Durkheim:

Lo hizo sobre la base del pensamiento de Saint Simón (1760-1825) y Augusto Comte (1798-1857), quienes, por otra parte, son lo que sentaron las bases del pensamiento sociológico... y verdaderos paradigmas del positivismo y pilares ideológicos del desarrollo del sistema educativo moderno. Sus ideas sobre la educación constituyen el primer antecedente, remoto, por cierto, de la sociología de la educación. Dicho, en otros términos, al germen de esta disciplina hay que buscarlo en el pensamiento positivo; consecuentemente, su orientación fue, en los orígenes positivista (p. 11).

Entre los autores que anteceden a los clásicos especialmente a Durkheim (18581917) esta Saint Simón (1760-1825) y Augusto Comte (1798-1857); así mismo en el siglo XX continúan con este pensamiento y ejercen gran influencia sobe las orientaciones actuales de la sociología de la educación Karl Mannheim (1893-1947) y Talcott Parsons (1902-1979). Desde este referente, se hace necesario abordar de manera general cuatro autores clásicos de la sociología de la Educación.

En la Tabla 6 se realiza un esbozo general de los representantes de la sociología clásica de la educación con el propósito de identificar y consolidar una posible comparación entre las escuelas que se han identificado. 
TABLA 6.

Representantes de la Sociología Clásica de la Educación

\begin{tabular}{cc}
\hline Autor & Breve descripción \\
\hline
\end{tabular}

Su perspectiva sociológica prioriza a las estructuras sociales por encima del individuo, le concede especial importancia a la socialización y a los procesos reproductivos de las pautas socioculturales, dando gran valor a la coacción y coerción social sobre el individuo, siendo la moral el elemento que permea todo el

El Funcionalismo y la influencia de Durkheim (1858-1917).

El individualismo metodológico:

Max Weber

(1864-1920).

La perspectiva marxista (1818-1883).

Los aportes e influencia de Simmel (1858-1918). proceso educativo. La socialización es de suma importancia, porque opera como el proceso de integración de los individuos a la sociedad y la adquisición por parte de estos de las competencias necesarias. El resultado final, es la dotación de un cierto modelo de individuo para una determinada sociedad en un espacio y tiempo definido.

Otros de los conceptos claves para entender la obra durkheniana son "acción general", "acción colectiva", "solidaridad mecánica", "solidaridad orgánica" y "hecho social", pero debido a la amplitud de estos, no es posible desarrollarlos ampliamente.

Esta corriente se relaciona en lo que algunos autores llaman sociología comprensiva, de la interacción social, individualismo metodológico o accionismo. La perspectiva teórica weberiana se centra en la acción social y la interacción social (en este caso lo social es el resultado de esta interacción). Como señala en Economía y Sociedad (Weber, 1922/2008) la sociología es "una ciencia que pretende entender, interpretándola, la acción social para de esa manera explicarla causalmente en su desarrollo y efectos".

La sociología debe encargarse de interpretar la acción a partir de la evidencia obtenida. Sin embargo, "la explicación interpretativa frente a la observación tiene ciertamente como precio el carácter esencialmente más hipotético y fragmentario de los resultados alcanzados por la interpretación. Pero es precisamente lo específico del conocimiento sociológico".

El tema de la educación no fue el centro de sus trabajos más representativos, el autor desarrolló algunos aspectos referentes a la educación, como en "El Capital (Tomo I), Trabajo de los niños y escolarización" (1867/2007) y en ciertos escritos como "Exámenes y Burocracia" (1843/2007), tomado de "La crítica de la filosofía del derecho" de Hegel, entre otros.

En el primero de ellos, señala la situación de explotación que sufren mujeres y niños en las fábricas y empresas. Ante tal situación el Parlamento inglés estableció como obligatoria la enseñanza hasta los 14 años, pero dicha ley no se cumplió. Lo anterior, junto con las condiciones de las escuelas es, para el autor, producto de la desprotección del Estado y la explotación burguesa. Sobre Exámenes y Burocracia, señala que la burocracia es una jerarquía del saber entre los distintos estratos de la pirámide del poder burocrático, lo cual no está al alcance de todos. En este punto, los exámenes se convierten en la entrada a los privilegios del círculo de la burocracia, restringidos sólo para quienes aprueben los exámenes.

Simmel trata de exponer su visión sobre el "arte de enseñar" y la educación, con base en la relación educación-enseñanza, los aspectos generales de la enseñanza y su aplicación en materias específicas como matemáticas, idiomas, historia, etc. Para Simmel la educación es un proceso para la vida y no sólo para la sociedad (como en el caso de Durkheim). Por tal motivo no visualiza al estudiante como un agente pasivo, sino todo lo contrario o, lo visualiza como un agente activo que al igual que el docente aspiran a alcanzar el conocimiento Para Simmel (2008) los contenidos deben presentarse bajo un método genético de perspectiva histórica y sistemática: de lo más simple a lo más complejo, donde el docente asume los contenidos como materia prima de un proceso de rendimiento individual y vivo, debido a que Simmel visualiza al estudiante como un agente activo del proceso de enseñanza y aprendizaje. 
La Sociología de la Educación como disciplina que emerge de la sociología como ciencia y las dos con sus respectivos estatus ontológico y epistemológico, conllevan a fortalecer el objeto de estudio desde una línea histórica, emergen los aportes de la sociología en el siglo XIX y la Sociología de la Educación de manera formal a mediados del siglo XX, sin descuidar los pensadores que antecedieron a los representantes clásicos, desde luego los clásicos y quienes en adelante han avanzado en este objeto de estudio.

Entre los enfoques de la Sociología de la Educación se puede señalar a Saint Simón (1760-1825) y Augusto Comte (1798-1857), que corresponden a los antecesores de los clásicos; posteriormente en el siglo XX ejercen gran influencia sobre las orientaciones actuales de la sociología de la educación Karl Mannheim (1893-1947) y Talcott Parsons (1902-1979) entre otros, pero por lo general derivan de las corrientes de Karl Marx (1818-1883), Max Weber (1864-1920) y Emile Durkheim (1858-1917).

Al respeto Feito (s. f.) expone:

Aunque seguramente se podrían detectar mayor número de marcos interpretativos de la educación desde la sociología, aquí nos centraremos en cinco grandes paradigmas clásicos: los enfoques funcionalistas (que arrancarían de Durkheim), los marxistas, los weberianos, los interpretativos y, por su imposible encasillamiento en uno u otro paradigma y la trascendental importancia de sus aportaciones, la obra de Bourdieu. Al considerar tan solo estos paradigmas clásicos, quedan fuera de esta introducción enfoques actuales como el post-modernismo, el feminismo, el multi-culturalismo y un largo etcétera (p. 15).

A lo largo del escrito de Feito (s. f.), destacan posturas paradigmáticas de donde derivan aportes significativos al desarrollo de la disciplina objeto de estudio; en este sentido, Brigido (2016) argumenta:

En la década de los ochenta, de la mano de los movimientos feministas de reivindicación de los derechos de la mujer, emerge uno de los temas descuidado por los enfoques establecidos en a sociológica de la educación: el del papel de la escuela en la reproducción de las relaciones de género (p. 74).

Consecuentemente, los retos y desafíos de la Sociología de la Educación se pueden enmarcar en tres ámbitos de trabajo: económico, político y cultural; con todas las implicaciones de rigor académico y científico de las cuales son objeto de estudio para los fines pertinentes de fortalecer el estatus de la disciplina.

La Sociología de la Educación ha de avanzar en los estudios sociales referidos a la educación como las teorías de la educación en el comienzo de un nuevo siglo, los nuevos paradigmas, las nuevas teorías, el grado de cientificidad del trabajo del educador y Teorías predominantes de la educación (Varas, 2003), son entre otros, los retos y desafíos de la educación y de la sociología de la educación en el análisis convergente de lo emergente en la educación y en la Sociología y como se puede dirimir para hacer una discusión se mayor alcance con implicaciones en las teorías formales en el campo de las Ciencias Sociales y Humanas. 
La importancia de la sociología de la educación, como se puede evidenciar desde las mismas generalidades expuestas al inicio del artículo, es consecuente entre si y se explica a lo largo del documento. Entre tanto, se analizan las etapas de la Sociología de la Educación de la segunda mitad del siglo XX desde la perspectiva teórica y la investigación de Bonal (1998) y en consecuencia es apropiada para abordar los planteamientos de Criado (2003). Para los autores, la sociología es ciencia que puede estudiar los hechos sociales, la verdadera ciencia de la educación es la sociología por cuanto sirve de fundamento para el origen de las ciencias de la educación.

\section{Consideraciones Finales}

Para estudiar los aportes de la Sociología a la Educación, es necesario considerar los nuevos enfoques de la sociología y tendencias disciplinares; es necesario analizar el desarrollo de la sociología como ciencia social, desde la época que se remonta a los autores clásicos hasta la actualidad; se puede observar que la comunidad científica sociológica ha mostrado un especial interés en la aplicación del conocimiento sociológico al estudio de la educación desde diversas perspectivas y el esbozo general de autores clásicos como Durkheim, Weber, Marx y Simmel.

Los aportes de la Sociología de la Educación a la formación de profesores, de manera especial a quienes se han preparado en el área de educación básica primaria y desempeñan su tarea profesional de manera genuina y autentica, se evidencian en nociones generales y bases especificas referidas a la relación entre la sociología y la educación como fundamento a la construcción de una sociedad divergente, donde se describen particularidades que emergen de las propias realidades de contexto desde las condiciones particulares y generales de los niños en la educación primaria.

\section{REFERENCIAS}

Adorno, T. y Horkheimer, M. (1969). La sociedad. Lecciones de sociología. Buenos Aires: Proteo.

Araque, J. y Rivera, N. (2005). Sociología General. Bogotá, D.C.: Trillas.

Austin, T. (s. f.). La socialización educativa vista por la sociología. (Sociología y Antropología de la socialización y la educación). [Online]. Disponible en http://www.robertexto.com/archivo10/socializ_educat.htm

Bernal, A. (2006). Antropología de la educación para la formación de profesores. Educación y Educadores, 9(2), 149-167. Disponible en https://educacionyeducadores.unisabana.edu.co/index.php/eye/article/view/668

Bonal, X. (1998). Sociología de la Educación: Una aproximación crítica a las corrientes contemporáneas. Barcelona: Paidós. 
Brigido, A. (2016). Sociología de la Educación: Temas y perspectivas fundamentales. Córdoba: Brujas.

Criado, E. (2003). Una crítica de la sociología de la educación crítica. Anduli. Revista Andaluza de Ciencias Sociales, (2), 9-27. Disponible en https://revistascientificas. us.es/index.php/anduli/article/view/3746

Feito, R. (s. f.). Teorías sociológicas de la educación. Madrid: Universidad Complutense de Madrid. Disponible en: http://pendientedemigracion.ucm.es/BUCM/cps/lecturas/4.htm

Parsons, T. (1976). El aula como un sistema social: algunas de sus funciones en la sociedad americana. re-Revista de Educación, (242), 64-86. Disponible en http://www. educacionyfp.gob.es/revista-de-educacion/numeros-revista-educacion/numeros-anteriores/1976/re242.html

Rodelo, M. y Castro, S. (2016). Reflexiones sobre la educabilidad: elemento fundamental en el proceso educacional del desarrollo humano. Cultura Educación y Sociedad, 7(2), 94-104. Disponible en https://revistascientificas.cuc.edu.co/culturaeducacionysociedad/article/view/1104

Rodríguez, A. (2011). Fundamentos de Sociología. (2 Ed.). Bogotá, D.C.: Ecoe.

Rojas-León, A. (2014). Aportes de la sociología al estudio de la educación (Autores lásicos). Revista Educación, 38(1), 33-58, enero-junio Disponible en http://revistas.ucr. ac.cr/index.php/educacion/article/view/14376/13680

Simmel, G. (2008). Pedagogía Escolar. Barcelona: Gedisa.

Varas, I. (2003). Tendencias predominantes de la educación contemporánea. Revista Investigación y Postgrado, 18(1), 46-57. Recuperado de https://bivir.uacj.mx/Reserva/ Documentos/rva2006133.pdf

*Artículo derivado de investigación. Es parte del proyecto titulado: Entre la didáctica de las ciencias humanas. y ciencias naturales: la perspectiva pedagógica conectivista en la formación de docentes, con código SGI 2683.

Jose Eriberto Cifuentes Medina es profesor de la Universidad Pedagógica y Tecnológica de Colombia (Tunja, Colombia). Licenciado en Filosofía y Educación Religiosa, Licenciado en Teología de la Universidad Santo Tomás (Bogotá , D.C., Colombia). Especialista en Educación con énfasis en Evaluación Educativa de la Universidad Santo Tomás (Bogotá, D.C., Colombia). Doctor en Educación de la Universidad de Baja California (Tepic, México). https://orcid.org/0000-0001-5702-620X

Nubia Elena Pineda De Cuadros es profesora de la Universidad Pedagógica y Tecnológica de Colombia (Tunja, Colombia). Licenciada en ciencias de la Educación de la Universidad Pedagógica y Tecnológica de Colombia-UPTC (Tunja, Colombia). Magister en Historia de la Universidad Pedagógica y Tecnológica de Colombia-UPTC (Tunja, Colombia). Doctora en Historia de la Universidad Pedagógica y Tecnológica de Colombia-UPTC (Tunja, Colombia). https://orcid.org/0000-0001-8646-257X 
Jaime Andres Torres Ortiz es docente e investigador en el área de Teoría y Práctica de la Investigación, con formación docente en programas de Licenciatura y Educación Superior, Uso de las TIC en la práctica pedagógica de la Universidad Pedagógica y Tecnológica de Colombia (Tunja , Colombia). Especialista en Gerencia de Recursos Humanos de la Universidad EAN (Bogotá, D.C., Colombia). Magister en Educación de la Universidad Javeriana (Bogotá, D.C., Colombia). Doctor en Ciencias de la Educación de la Universidad Pedagógica y Tecnológica de Colombia-UPTC (Tunja, Colombia). https://orcid.org/00000003-3720-2960 